\title{
Perfil Antropométrico y Composición Corporal de la Selección Mexicana Varonil Mayor de Voleibol
}

\author{
Anthropometric Profile and Body Composition of the Mexican Men's Volleyball Team
}

Lenin Tlamatini Barajas Pineda ${ }^{1}$; Ciria Margarita Salazar C. ${ }^{1}$; José E. Del Río Valdivia ${ }^{1}$;

Pedro Julián Flores Morenoㅜ; Julio Alejandro Gómez Figueroa² \& Eduardo Gómez Gómez ${ }^{1}$

\begin{abstract}
BARAJAS, P. L. T.; SAlazAR, C. C. M.; DEL RÍO, V. J. E.; FLORES, M. P. J.; GÓMEZ, F. J. A. \& GÓMEZ, G. E. Perfil antropométrico y composición corporal de la selección mexicana varonil mayor de voleibol. Int. J. Morphol., 39(1):90-94, 2021.

RESUMEN: Determinar el perfil antropométrico y de composición corporal de los atletas ha sido — hasta hace poco— una herramienta fundamental para predecir el rendimiento deportivo y comportamiento biomecánico. Para este estudio, se evaluaron a 15 jugadores de la Selección Mexicana de Voleibol Varonil, con edad de 26,4 4 4,73, previo a la XIV Copa Panamericana de Voleibol 2019. Se utilizó el protocolo ISAK para la toma antropométrica así como las ecuaciones de Carter \& Heath para determinar el perfil antropométrico y somatotipo, el porcentajey kg de grasa, se calculó a partir de la ecuación de Durnin \& Womersley, la masa muscular con el método De Rose y Gimares, y tejido óseo con la ecuación de Rocha. Resultados: el peso promedio fue de 85,45 kg ( $\pm 8,39)$, estatura 192,71 cm ( \pm $7,15)$; somatotipo: endomorfo $(2,3 \pm 0,8)$, mesomorfo $(4,0 \pm 1,4)$ y ectomorfo $(3,6 \pm 1,5)$. El \% GC promedio fue de $(3,6 \pm 1,5)$, kg GC $(11,2 \pm 4,0)$, de \% MM (43,8 $\pm 2,3), \operatorname{kg}$ MM $(37,3 \pm 2,7), \%$ TO $(19,3 \pm 1,8), \operatorname{KgTO}(16,4 \pm 1,2)$. Se concluye que la Selección Mexicana Varonil Mayor de Voleibol se encuentra dentro de los rangos competitivos de una Selección mayor; así como también muestra valores de referencia internacional. Presenta dos perfiles somatotípicos, endo-mesomórfico para jugadores defensivos y ecto-mesomórficos en jugadores ofensivos, beneficiando técnicamente su desempeño.
\end{abstract}

PALABRAS CLAVE: Somatotipo; Composición corporal; Antropometría; Voleibol.

\section{INTRODUCCIÓN}

El voleibol tiene una clasificación praxiológica que lo define como un deporte de cooperación-oposición (Hernández, 2000), disciplina rápida y de gran habilidad coordinativa (Ciccarone et al., 2008), su estructura y dinámica de juego se divide en dos apartados:el ataque y la defensa del ataque. Rentero \& Moreno (2015) mencionan que la ofensiva y defensa, están ligadas al ataque; de ahí su alta exigencia física y técnica, gestos motrices de saltos y movimientos explosivos; además, un rasgo antropométrico (como la estatura superior al promedio). Se considera factor determinante para el rendimiento óptimo (Durnin \& Womersley, 1974; Almagià et al., 2009).

La Federación Internacional de Voleibol (FIVB) ha modificado las reglas, previendo la transformación del performance deportivo; algunos de los cambios son los del fortalecimiento de la defensa con la presencia del líbero y en el ataque con el sistema de puntuación Rally Point
System (Rentero \& Moreno), que han permitido un mayor dinamismo; y, por consecuencia, una mayor exigencia física, psicológica y de respuestas tácticas. Otros cambios del reglamento responden a la evolución morfológica de la estatura absoluta debido a que hay una tendencia al incremento a nivel mundial (Norton et al., 2000).

Por lo anterior, la antropometría toma importancia, ya que ayuda a definir los rasgos morfológicos de los atletas; con la oportunidad de evaluar y determinar la composición corporal y el somatotipo (Almagià et al.), existen equipos internacionales — como el de Rusia, Brasil, Estados Unidos y Cuba- donde se identifican y evalúan las características antropométricas para aumentar el rendimiento del jugador, según su somatotipo y posición de juego (Lentini et al., 2006); dichos parámetros, han sido valorados a nivel mundial (Papadopoulou, 2001; Almagià et al.), obteniendo datos de referencia, a través de los cuales se puede predecir la oportunidad de éxito.

\footnotetext{
${ }^{1}$ Universidad de Colima, Colima, México.

${ }^{2}$ Universidad Veracruzana, Veracruz, México.
} 
Con el paso de los años se han incorporado variables de estudio—como lo es el tejido muscular, óseo y grasoempleados para determinar el estado nutricional y el nivel de rendimiento físico (De Hoyo et al., 2008; Almagià et al.; Fonseca et al., 2010; Pérez et al., 2014; Muñoz-Marín et al., 2016; Zamora, 2018; López et al., 2019).

En México,se tiene como antecedente los estudios en jugadores de voleibol de Rivera (2016), de Brizuela (2016) y López et al.; en donde se determinan perfiles antropométricos, de somatotipo y composición corporal en Selectivos Universitarios, tal como lo sugieren Lentini et al.; pues, afirman, que en el deporte "la antropometría surge como herramienta para el desarrollo del alto rendimiento para cuantificar las formas corporales y dimensiones, convirtiéndose en un método valioso en el área de las mediciones corporales de los deportistas". El presente estudio (pionero) se realizó en jugadores de la Selección Mexicana Varonil Mayor de Voleibol de Sala; siendo referente para realizar investigación comparada con la elite del voleibol mexicano.

\section{MATERIAL Y MÉTODO}

La investigación fue de tipo descriptivo transversal (Morales, 2012). La población de estudio se conformó por 15 jugadores de la Selección Mexicana Varonil Mayor de Voleibol (edición 2019); conformada por 15 jugadores con edad promedio en años de $26,4 \pm 4,73$, a los cuales se les administró el método antropométrico del somatotipo de Carter \& Heath (1990), caracterizado por ser un método tricompartimental que se dividió la morfo-estructura en masa muscular, masa ósea y masa magra. Para ello, se usó el protocolo del perfil restringido y el kit antropométrico Rosscraft SRL, validado por la Asociación Internacional de Kinantopometría (ISAK), que se compone por paquímetro óseo, cinta métrica y plicómetro. Los datos se recabaron por personal certificado en nivel II de ISAK y en condiciones normales de temperatura, por la mañana y después del vacío urinario; previo a las evaluaciones se consideró el acuerdo a la Declaración Helsinki: se informó a los jugadores sobre las tomas, implicaciones y efectos secundarios derivados del protocolo (Manzini, 2000).

Los valores se obtuvieron derivados de evaluaciones previas a la etapa competitiva de la Copa Panamericana 2019 —desarrollada en Colima, México- en donde participaron 12 países adheridos a la Confederación de Voleibol de América Central y el Caribe (NORCECA); por lo que, en consideración del tiempo durante el cual se intervino, la Selección Nacional se encontraba en estado de rendimiento máximo. Los datos fueron registrados en la plantilla de recolección manual y, posteriormente (para su traducción) en plantilla de Excel. Se emplearon técnicas estadísticas de media y desviación estándar para describir a la población y la prueba de Kruskall Wallis para identificar diferencias entre los grupos. Para ello, se utilizó el paquete SPSS, versión 22.

\section{RESULTADOS}

En la Tabla I, se observan los valores antropométricos básicos, así como la edad: 26,40 \pm 4,73 años; peso $85,45 \pm$ $8,39 \mathrm{~kg}$; estatura 192,71 \pm 7,16 cm; Índice de Masa Corporal 23,07 $\pm 2,44$; en los cuales no existen diferencias estadísticas significativas entre las variables mencionadas por posiciones de juego.

En la Tabla II se presenta la estadística descriptiva de pliegues, circunferencias y diámetros por posiciones de juego. No se encontraron diferencias estadísticamente significativas.

En la composición corporal, quienes presentan mayor porcentaje de grasa son los acomodadores $(19,46 \pm 3,51)$, los banda poseen mayor porcentaje de Masa Muscular (45,70 $\pm 1,28)$; los centrales presentan mayor porcentaje de peso óseo $(20,52 \pm 2,74)$ y la presencia de diferencias significativas entre posiciones en la variable de porcentaje de Masa Muscular ( $\mathrm{p}<0,05=0,033$ ) (Tabla III).

Tabla I. Descriptivos por posición de juego de peso, estatura e IMC ( \pm sd) de la Selección Mexicana Varonil Mayor de Vóleibol.

\begin{tabular}{llllll}
\hline Posición & N & \multicolumn{1}{c}{ Edad } & \multicolumn{1}{c}{ Peso $(\mathrm{kg})$} & \multicolumn{1}{c}{ Estatura $(\mathrm{cm})$} & IMC \\
\hline Banda & 5 & $26,60 \pm 2,88$ & $83,18 \pm 4,54$ & $189,10 \pm 6,74$ & $23,32 \pm 1,92$ \\
Acomodador & 2 & $26,50 \pm 6,36$ & $87,00 \pm 13,43$ & $189,95 \pm 3,041$ & $24,10 \pm 2,96$ \\
Central & 4 & $24,50 \pm 3,69$ & $88,82 \pm 13,61$ & $200,95 \pm 6,26$ & $22,07 \pm 3,734$ \\
Líbero & 2 & $31,50 \pm 10,60$ & $88,40 \pm 3,39$ & $188,30 \pm 3,81$ & $24,05 \pm 0,070$ \\
Opuesto & 2 & $24,50 \pm 3,53$ & $79,90 \pm 1,13$ & $192,55 \pm 1,48$ & $21,55 \pm 0,63$ \\
Promedio general & & $26,40 \pm 4,73$ & $85,45 \pm 8,39$ & $192,71 \pm 7,16$ & $23,07 \pm 2,44$ \\
p valor & & 0,795 & 0,758 & 0,087 & 0,482 \\
\hline
\end{tabular}

$\mathrm{N}=$ Número de sujetos, $\mathrm{p}$ valor $=$ Kruskal Wallis . 
Tabla II. Estadística descriptiva de pliegues, circunferencias y diámetros ( $\mathrm{x} \pm \mathrm{sd})$.

\begin{tabular}{|c|c|c|c|c|c|c|c|}
\hline \multicolumn{8}{|c|}{ Posiciones de juego } \\
\hline Parámetro & Banda $(5)^{*}$ & $\begin{array}{c}\text { Acomodador } \\
(2)^{*}\end{array}$ & Central $(4)^{*}$ & Líbero (2)* & Opuesto (2)* & PG & $\mathrm{p}$, valor \\
\hline P, Tríceps (mm) & $6,40 \pm 2,30$ & $9,00 \pm 1,41$ & $9,87 \pm 8,81$ & $8,15 \pm 1,20$ & $6,25 \pm 1,76$ & $7,88 \pm 4,59$ & 0,499 \\
\hline $\mathrm{P}$, Subescapular $(\mathrm{mm})$ & $9,10 \pm 1,34$ & $13,65 \pm 4,73$ & $10,12 \pm 3,27$ & $11,15 \pm 0,21$ & $8,25 \pm 0,35$ & $10,14 \pm 2,68$ & 0,188 \\
\hline P, Bíceps (mm) & $2,40 \pm 0,41$ & $3,25 \pm 1,06$ & $4,25 \pm 2,53$ & $3,00 \pm 0,00$ & $3,50 \pm 0,00$ & $3,23 \pm 1,43$ & 0,154 \\
\hline P, Cresta ilíaca (mm) & $11,92 \pm 2,54$ & $18,50 \pm 3,53$ & $15,62 \pm 9,01$ & $19,50 \pm 2,12$ & $10,90 \pm 1,97$ & $14,66 \pm 5,58$ & 0,252 \\
\hline P, Supra espinal (mm) & $8,0 \pm 2,3$ & $9,25 \pm 1,06$ & $7,95 \pm 3,09$ & $12,75 \pm 1,76$ & $6,00 \pm 0,00$ & $8,52 \pm 2,76$ & 0,199 \\
\hline P, Abdominal (mm) & $14,20 \pm 4,77$ & $16,00 \pm 5,65$ & $18,00 \pm 9,06$ & $27,00 \pm 2,82$ & $13,40 \pm 1,27$ & $17,05 \pm 6,81$ & 0,326 \\
\hline P, Muslo Frontal (mm) & $7,90 \pm 2,92$ & $12,500 \pm 3,53$ & $10,62 \pm 6,65$ & $9,15 \pm 4,45$ & $8,25 \pm 1,06$ & $9,45 \pm 4,13$ & 0,660 \\
\hline P, Pantorrilla Med (mm) & $4,70 \pm 1,25$ & $5,50 \pm 0,70$ & $6,00 \pm 4,77$ & $5,00 \pm 1,41$ & $5,50 \pm 2,12$ & $5,30 \pm 2,47$ & 0,882 \\
\hline C, Brazo Relaja do $(\mathrm{cm})$ & $33,60 \pm 1,61$ & $34,25 \pm 3,74$ & $33,72 \pm 4,72$ & $35,50 \pm 0,70$ & $32,50 \pm 1,55$ & $33,84 \pm 2,72$ & 0,599 \\
\hline $\mathrm{C}$, Brazo Contraído $(\mathrm{cm})$ & $36,28 \pm 1,92$ & $36,30 \pm 3,81$ & $35,87 \pm 2,80$ & $37,90 \pm 1,41$ & $34,60 \pm 2,82$ & $36,16 \pm 2,30$ & 0,561 \\
\hline $\mathrm{C}$, Cintura $(\mathrm{cm})$ & $82,10 \pm 3,50$ & $85,25 \pm 8,83$ & $82,85 \pm 6,68$ & $88,60 \pm 2,40$ & $81,70 \pm 2,54$ & $83,53 \pm 4,99$ & 0,555 \\
\hline $\mathrm{C}$, Cadera $(\mathrm{cm})$ & $101,10 \pm 5,36$ & $102,25 \pm 4,59$ & $102,75 \pm 7,62$ & $105,20 \pm 1,13$ & $98,50 \pm 1,27$ & $101,89 \pm 5,10$ & 0,866 \\
\hline C, Pantorrilla $(\mathrm{cm})$ & $37,08 \pm 2,26$ & $37,20 \pm 0,28$ & $36,40 \pm 2,34$ & $39,00 \pm 1,41$ & $36,00 \pm 0,98$ & $37,02 \pm 1,91$ & 0,346 \\
\hline $\mathrm{D}$, Húmero $(\mathrm{cm})$ & $7,26 \pm 0,31$ & $7,25 \pm 0,35$ & $7,50 \pm 0,43$ & $7,25 \pm 0,07$ & $7,40 \pm 0,14$ & $7,34 \pm 0,30$ & 0,751 \\
\hline $\mathrm{D}$, Fémur $(\mathrm{cm})$ & $9,76 \pm 0,55$ & $9,8 \pm 0,49$ & $10,12 \pm 0,34$ & $10,15 \pm 0,07$ & $9,45 \pm 0,07$ & $9,88 \pm 0,43$ & 0,285 \\
\hline
\end{tabular}

$(\#)^{*}=$ Cantidad de jugadores por posición de juego, $\mathrm{x} \pm \mathrm{sd}=$ Promedio \pm Desviación Estándar, $\mathrm{PG}=$ Promedio General, $\mathrm{p}$ valor $=$ Kruskal Wallis.

Tabla III. Estadísticos descriptivos de kg y \% de Grasa Corporal, Masa Magra y Masa Ósea. (x \pm sd).

\begin{tabular}{lccccccc}
\hline Posiciones & N & Kg GC & \% GC & Kg MM & \% MM & Kg O & \% O \\
\hline Banda & 5 & $9,48 \pm 1,40$ & $13,79 \pm 2,86$ & $38,04 \pm 2,58$ & $45,70 \pm 1,28$ & $15,70 \pm 0,79$ & $18,92 \pm 1,19$ \\
Acomodador & 2 & $13,30 \pm 4,8$ & $19,46 \pm 3,51$ & $37,05 \pm 5,02$ & $42,65 \pm 0,77$ & $15,85 \pm 0,35$ & $18,45 \pm 2,47$ \\
Central & 4 & $12,52 \pm 6,82$ & $16,69 \pm 7,35$ & $36,97 \pm 3,43$ & $41,95 \pm 2,84$ & $17,97 \pm 0,96$ & $20,52 \pm 2,74$ \\
Líbero & 2 & $13,30 \pm 0,14$ & $18,79 \pm 0,38$ & $37,85 \pm 1,76$ & $42,80 \pm 0,42$ & $16,05 \pm 0,63$ & $18,15 \pm 0,07$ \\
Opuesto & 2 & $8,90 \pm 0,56$ & $13,52 \pm 1,65$ & $35,85 \pm 0,35$ & $44,85 \pm 0,21$ & $15,95 \pm 0,07$ & $19,95 \pm 0,35$ \\
\multicolumn{2}{r}{ Promedio General } & $11,220 \pm 3,96$ & $15,95 \pm 4,54$ & $37,30 \pm 2,65$ & $43,79 \pm 2,23$ & $16,40 \pm 1,17$ & $19,32 \pm 1,81$ \\
& $\boldsymbol{p}$ valor & $\mathbf{0 , 3 4 1}$ & $\mathbf{0 , 2 4 2}$ & $\mathbf{0 , 7 5 9}$ & $\mathbf{0 , 0 3 3 *}$ & $\mathbf{0 , 0 7 8}$ & $\mathbf{0 , 4 0 4}$ \\
\hline
\end{tabular}

$\mathrm{x} \pm \mathrm{sd}=$ Promedio y Desviación Estándar, Kg GC = Kilogramo de Grasa Corporal, \% GC = Porcentaje de Grasa Corporal, Kg MM = Kilogramo de Masa Magra, \% MM = Porcentaje de Masa Magra, Kg O = Kilogramos de Tejido Óseo, \% O = Porcentaje de Tejido Óseo, p valor = Kruskal Wallis.

El promedio del componente endomórfico es de $2,3 \pm 0,8$ ( $\mathrm{p}>0,05=, 220)$, del mesomórfico es de 4,0 $\pm 1,3$ $(\mathrm{p}>0,05=, 238)$, y del ectomórfico de 3,5 $\pm 1,4(\mathrm{p}>0,05=$ ,307). En ninguno de los componentes se presentaron diferencias estadísticas significativas por posición de juego (Tabla IV).
Si se ubican estos valores en la somato-carta se podría observar la dispersión de los componentes mesomorfo y sus derivados; en promedio, los jugadores banda son mesoectomórfico, mientras que los acomodadores, en mesomorfo balanceado; los centrales y opuestos en ecto-mesomorfos, mientras que los líberos en meso-endomorfos; la Selección se localiza, en lo general, en ecto-mesomorfo.

Tabla IV. Estadísticos de los componentes endomorfia, mesomorfia y ectomorfia,(x $\pm \mathrm{sd})$.

\begin{tabular}{lcccl}
\hline Posiciones & Endomorfia & Mesomorfia & Ectomorfia & Escala \\
\hline Banda & $2,0 \pm 0,4$ & $4,4 \pm 1,2$ & $3,8 \pm 1,1$ & Meso-Ectomorfo \\
Acomodador & $2,9 \pm 0,7$ & $4,3 \pm 0,2$ & $2,9 \pm 1,1$ & Mesomorfo Bala nceado \\
Central & $2,2 \pm 1,3$ & $3,0 \pm 1,7$ & $4,5 \pm 2,2$ & Ecto-Mesomorfo \\
Líbero & $2,9 \pm 0,7$ & $5,3 \pm 0,2$ & $2,4 \pm 0,2$ & Meso-Endomorfo \\
Opuesto & $1,7 \pm 0,1$ & $3,4 \pm 0,8$ & $4,2 \pm 0,4$ & Ecto-Mesomorfo \\
Promedio general & $2,3 \pm 0,8$ & $4,0 \pm 1,3$ & $3,5 \pm 1,4$ & Ecto-Mesomorfo \\
\hline $\boldsymbol{p}$ valor & $\mathbf{0 , 2 2 0}$ & $\mathbf{0 , 2 3 8}$ & $\mathbf{0 , 3 0 7}$ & \\
\hline
\end{tabular}

$\mathrm{x} \pm \mathrm{sd}=$ Promedio y Desviación Estándar. 


\section{DISCUSIÓN}

López et al. hacen énfasis en lo fundamental que es el control corporal del deportista para la formación y desarrollo del rendimiento; por ello, los resultados son de relevancia para comparar los hallazgos con los de otros selectivos nacionales e internacionales, como es el caso de la Competencia Panamericana; donde el promedio de edad fue de $24,51 \pm 1,40$, una diferencia promedio de 1,89 años con el selectivo mexicano, donde los de mayor edad son los jugadores de posición líbero (con 31,50 $\pm 10,60$ ). Estos jugadores de acción defensiva son los de mayor experiencia, tal como lo cita Rentero \& Moreno. En cuanto al peso y talla promedio entre las selecciones fue de 85,29 \pm 9,11; mientras que la Selección Mexicana fue de $85,45 \pm 8,39$ y $192,71 \pm 7,16$, contra el promedio general de las selecciones, $191 \pm 0,04$, respectivamente. El Índice de Masa Corporal de las selecciones en promedio fue de 23,59 $\pm 2,64$, comparado con 23,07 $\pm 2,44$ del selectivo mexicano; precisando que todos los participantes se encuentran en el rango de normo-peso, según los criterios de la Organización Mundial de la Salud.

A diferencia del estudio realizado por García-Alcaraz et al. (2014), quienes indican que el rendimiento de la defensa y la ofensiva está condicionado por las características antropométricas del jugador; destacan las variables relacionadas con la superficie de contacto (anchura de hombros, extensión de codos, anchura de antebrazos, etc.); y en la ofensiva, la altura del jugador, que es determinante para el rendimiento de acciones que se realizan en salto (saque, ataque y bloqueo). Los seleccionados nacionales no mostraron esta condición, al no haber diferencias significativas en los parámetros evaluados con el perfil restringido; aunque dichas condiciones pueden ser modificables posterior a 135 días de entrenamiento específico por posiciones de juego (Prudêncio $\&$ Tumelero, 2006).

Se destaca que el porcentaje de Masa Muscular entre las diferentes posiciones de juego mostró diferencia significativa ( $p>0,05=0,033$ ), superior en los jugadores que ocupan la posición de banda $(45,70 \pm 1,28)$, menor en los centrales. Estos hallazgos coinciden con la precisión realizada por García-Alcaraz et al. sobre las características de aptitud físicas y antropométricas de los ofensivos, por el contrario, de los defensivos.

Al comparar los resultados del presente estudio con los encontrados en selectivos universitarios (López et al.; Rivera; Brizuela), la Selección Mexicana 2019, presenta menor porcentaje tanto de grasa como de Masa Muscular, excepto con los datos del estudio de Brizuela. Al realizar la comparación con estudios de selecciones internacionales, la Selección
Mexicana presenta menores niveles de porcentaje tanto de grasa corporal como de masa muscular que los representativos de Chile, Colombia, Paraguay y Venezuela (Almagià et al.), con relación a los componentes promedio del somatotipo. La Selección Mexicana obtuvo en endomorfia de 2,3 $\pm 0,82$; que implica, bajo desarrollo muscular relativo, endomorfia 4,0 \pm 1,35 , traducido como moderada adiposidad relativa y ectomorfia $3,5 \pm 1,49$, que se entiende como linealidad relativa moderada, en atención a la escala de Carter \& Heath; por lo que el biotipo promedio total de los jugadores esectomesomorfo.

Con relación a la bibliografía nacional, el somatotipo de los jugadores de voleibol universitario desarrollado por López et al., no hay diferencia en el componente endomórfico y ectomorfo, según lo presentado por Rivera; y con la bibliografía internacional, el somatotipo de la Selección Mexicana es diferente a la del biotipo cubano, dado que Carvajal \& Serviat (2014), indican que los promedios históricos (1970 a 2012) fue de endomorfia $(1,9 \pm 0,7)$, mesomorfia $(4,5 \pm 1,0)$ y ectomorfia $(3,3 \pm 1,0)$; es decir, menor grasa corporal, mayor masa muscular y similitud en linealidad que la Selección Mexicana. En este sentido, si comparamos el biotipo por posiciones de juego con juveniles brasileños, son diferentes; pues, en promedio, son ectomorfos balanceados que ectomesomorfos. Es decir, presentan menor desarrollo de masa muscular, sin embargo, destacamos que son jugadores de 16,8 $\pm 0,58$ años (Fonseca et al.) y sólo se encuentra similitud con los jugadores élite de Sevilla, en el componente mesomórfico (Fernández \& Alvero, 2006).

\section{CONCLUSIONES}

Es importante mencionar que los acomodadores y líberos presentaron el mayor porcentaje de grasa corporal; en tal sentido, consideramos que en estos jugadores deberán atendersemedidas nutricionales y de entrenamiento, con mayor énfasis en capacidad aeróbica.

Los resultados permiten concluir que los rangos competitivos son aceptables; ya que, en todas las variables analizadas se mantiene con promedios similares, en comparación con los representativos internacionales de nivel panamericano.

Es necesario integrar —en estos selectivos nacionales- equipos multidisciplinarios (nutriólogos, fisiólogos, médicos, psicólogos, etc.), que consulten los resultados y le den seguimiento para mejorar los somatotipos de nuestros representativos nacionales; ya que se debe pensar en la idoneidad de dos perfiles somato-típicos, endo-mesomórfico para jugadores defensivos; y ecto-mesomórficos para los ofensivos. Se 
requiere más información de carácter nacional e internacional que permita ubicar un biotipo de jugador por posición de juego; por lo que estos datos contribuirán a dicho objetivo, que se contempla como un área de oportunidad para futuras investigaciones.

BARAJAS, P. L. T.; SALAZAR, C. C. M.; DEL RÍO, V. J. E.; GÓMEZ, G. E.; GÓMEZ, F. J. A. \& FLORES, M. P. J. Anthropometric profile and body composition of the Mexican men's volleyball team Int. J. Morphol., 39(1):90-94, 2021.

SUMMARY: Until recently, determining the anthropometric and body composition profile of athletes has been a fundamental tool for predicting sports performance and biomechanical behavior. For this study, 15 players form the Mexican Men`s Volleyball Team, aged $26.4 \pm 4.73$, were evaluated prior to the beginning of the XIV 2019 PanAmerican Volleyball Cup, using the ISAK protocol. As well as the Carter $\&$ Heath equation to determine the anthropometric profile and somatotype, the percentage and $\mathrm{Kg}$ of fat, with the Durnin \& Womersley equation, the De Rose and Gimares muscle mass, and bone tissue with the Rocha equation. The average weight was $85.45 \mathrm{~kg}( \pm 8.39)$, height $192.71 \mathrm{~cm}( \pm 7.15)$; somatotype: endomorphic $(2.3 \pm 0.8)$, mesomorphic $(4.0 \pm 1.4)$, and ectomorphic (3.6 \pm 1.5$)$. The overage $\% \mathrm{GC}$ was $(3.6 \pm$ 1.5). kg GC (11.2 \pm 4.0$)$, de \% MM (43.8 \pm 2.3$) \mathrm{kg} \mathrm{MM}(37.3 \pm 2.7), \%$ TO $(19.3 \pm 1.8), \mathrm{kg}$ TO $(16.4 \pm 1.2)$. It is concluded that the Mexican Men`s Volleyball Team is within the competitive ranges of a larger international teams, in addition, and as international reference values, the Selection presents two somatotypes profile, endo-mesomorphic for defensive players and ecto-mesomorphic for offensive players, technically benefiting their performance.

KEY WORDS: Somatotype; Body composition; Anthropometric; Volleyball.

\section{REFERENCIAS BIBLIOGRÁFICAS}

Almagià, F. A. A.; Rodríguez, R. F.; Barraza, G. F. O.; Lizana, A. P. J.; Ivanovic, M. D. \& Binvignat, G. O. Anthropometric profile of professional volleyball sudamerican players. Int. J. Morphol., 27(1):53-7, 2009.

Brizuela O. M. Perfil antropométrico y aptitud física en voleibolistas juveniles de colima. Rev. Mex. Investig. Cult. Fís. Deporte, 7(9):217-30, 2016.

Carter, J. \& Heath, B. Somatotyping-Development and Applications. Cambridge, Cambridge University Press, 1990

Carvajal, V. W. \& Serviat, H. N. Evolución del somatotipo de jugadores cubanos de voleibol en el periodo 1970-2012. Arch. Med. Deporte, 31(6):384-90, 2014.

Ciccarone, G.; Croisier, J. L.; Fontani, G.; Martelli, G.; Albert, A.; Zhang, L. \& Cloes, M. Comparison between player specialization, anthropometric characteristics and jumping ability in top-level volleyball players. Med. Sporte, 61(1):29-43, 2008 .

De Hoyo, M.; Sañudo, B. \& Carrasco, L. Composición corporal y prevalencia de sobrepeso en jóvenes jugadores de voleibol. Rev. Int. Med. Cienc. Activ. Fís. Deporte, 8(32):256-69, 2008.

Durnin, J. V. \& Womersley, J. Body fat assessed from total body density and its estimation from skinfold thickness: measurements on 481 men and women aged from 16 to 72 years. Br. J. Nutr., 32(1):77-97, 1974.

Fernández, P. S. \& Alvero, C. J. R. La producción científica en cineantropometría: datos de referencia de composición corporal y somatotipo.Arch. Med. Deporte, Vol. 23 (111):17-35, 2006.

Fonseca, C. L. T ; Roquetti Fernandes, P. \& Fernandes Filho, J. Analysis of anthropometrical profile of Brazilian junior volleyball team. Int. J. Morphol., 28(4):1035-41, 2010.
García-Alcaraz, A.; Palao, J. M. \& Ortega, E. Perfil de rendimiento técnico-táctico de la recepción en función de la categoría de competición en voleibol masculino. Kronos, 13(1), 2014. Disponible en: https://g-se.com/perfil-de-rendimiento-tecnico-tactico-de-la-recepcion-en-funcion-de-la-categoria-decompeticion-en-voleibol-masculino-1706-sa-x $57 \mathrm{cfb} 272429 \mathrm{db}$

Hernández, M. J. La Iniciación a los Deportes desde su Estructura y Dinámica. Barcelona, INDE Publicaciones, 2000.

Lentini, N. A.; Cardey, M. L.; Aquilino, G. \& Dolce, P. A. Estudio Somatotípico en Deportistas de Alto Rendimiento de Argentina. PubliCE, 2006. Disponible en: https://g-se.com/estudio-somatotipico-en-deportistas-de-alto-rendimientode-argentina-738-sa-D57cfb2717d0b4

López, G. R.; Lagunes Carrasco, J. O.; Carranza García, L. E. \& Banda Sauceda, N. C. Características antropométricas en jugadores de voleibol universitario mexicano. Emás F Rev. Digit. Educ. Fís., (60):127-35, 2019.

Manzini, J. L. Declaración de Helsinki: principios éticos para la investigación médica sobre sujetos humanos. Análisis de la $5^{\mathrm{a}}$ reforma, aprobada por la Asamblea General de la Asociación Médica Mundial en octubre del año 2000, en Edimburgo*, respecto del texto aprobado en Somerset West (Sudáfrica) en octubre de 1996. Acta Bioeth., 6(2):321-34, 2000.

Morales, F. Conozca 3 tipos de investigación: Descriptiva, Exploratoria y Explicativa. Sitio Web. Creadess, Cooperación en Red Euro Americana para el Desarrollo Sostenible, 2012. Disponible en: http://www.creadess.org/ index.php/informate/de-interes/temas-de-interes/17300-conozca-3-tipos-deinvestigacion-descriptiva-exploratoria-y-explicativa

Muñoz-Marín, D.; Crespo Coco, M. C.; Grijota Pérez, F. J.; Iglesias Sánchez, P. J. \& Robles Gil, M. C. Evaluación antropométrica y de condición física en jugadores de voleibol en edad escolar. Diferencias entre sexos. Ágora Educ. Fís. Deporte, 18(1):77-88, 2016.

Norton, K.; Olds, T.; Craig, N. \& Olive, S. Antropometría y Performance Deportiva. En: Norton, K.; Olds, T. \& Mazza, J. C. (Eds.). Antropometrica. Rosario, Biosystem, 2000.

Papadopoulou, S. D. Anthropometric Characteristics of Greek Top Volleyball Players. The Effect of Demographic and Socioeconomic Factors on the Differentiation between Competition Levels. Disertación de Doctoado. Salónica, Department of Sport Medicine, Faculty of Physical Education and Sport Science, Aristotle University of Thessaloniki, 2001.

Pérez V. Y.; Portela Pozo, Y.; Cantero Márquez, M. \& Rodríguez Stiven, E. Características antropométricas de jugadores de voleibol en la universidad de la ciencias informáticas. Emás F Rev. Digit. Educ. Fís., (30):8-18, 2014.

Prudêncio, V. \& Tumelero, S. Capacidades físicas e de treinamento para diferentes posições das praticantes da modalidade de voleibol.efdeportes, 10(94), 2006 Disponible en: https://www.efdeportes.com/efd94/volei.htm

Rentero, L. \& Moreno, M. P. Análisis de la influencia del líbero en diferentes fases del juego en voleibol. Rev. Int. Med. Cienc. Activ. Fís. Deporte, 15(60):739$56,2015$.

Rivera, S. Análisis cineantropométrico de las selecciones nacionales universitarias de baloncesto y voleibol de México. Rev. Mex. Investig. Cult. Fís. Deporte, 7(9):141-56, 2016

Zamora, S. Actividad Física, Composición Corporal y Somatotipo de Estudiantes Universitarios. Conferencia. San José de Costa Rica, XXIV Simposio Internacional en Ciencias del Deporte, el Ejercicio y la Salud, Universidad de Costa Rica, Facultad de Educación, 2018.

Dirección para correspondencia:

Mtro. Pedro Julián Flores Moreno

Profesor Investigador de Tiempo Completo

Miembro del Cuerpo Académico UCol-CA 101 Cultura Física

Facultad de Ciencias de la Educación

Universidad de Colima

MÉXICO

Email: pedrojulian_flores@ucol.mx

Recibido : 18-07-2020

Aceptado: 25-09-2020 